\title{
Providing a Cloud Broker-based Approach to Improve the Energy Consumption and Achieve a Green Cloud Computing
}

\author{
Tina Samizadeh Nikoui \\ Department of Computer \\ Engineering, \\ Tehran North Branch, Islamic \\ Azad University, \\ Tehran, Iran
}

\author{
Sam Jabbehdari \\ Department of Computer \\ Engineering, \\ Tehran North Branch, Islamic \\ Azad University, \\ Tehran, Iran
}

\author{
Alireza Bagheri \\ Department of Computer \\ Engineering, \\ Tehran North Branch, \\ Islamic Azad University, \\ Tehran, Iran \\ Department of Computer \\ Engineering and Information \\ Technology, \\ Amirkabir University of \\ Technology, \\ Tehran, Iran
}

\begin{abstract}
Today`s, cloud computing has been widely accepted in the industry. With the increasing popularity of cloud computing, many users and companies want to use and offer cloud computing services. The growth of cloud computing services may lead to consume a huge amount of energy and emit considerable amount of carbon dioxide. In recent years raising concerns about global warming and environment impact of greenhouse gases emission has led many researchers to engage in research in the field of green and energy aware computing. In this paper, "two phase carbon aware cloud broker" has been proposed that attempt to minimize energy and carbon by considering the energy and carbon efficiency of data centers.
\end{abstract}

\section{General Terms}

Cloud computing, carbon emissions, energy aware.

\section{Keywords}

Cloud computing, carbon emissions, energy aware.

\section{INTRODUCTION}

Cloud computing delivers an infrastructure, platform and software as services that are made available to consumers in a pay-as-you-go model. In industry, these services are referred to as Infrastructure as a Service (IaaS), Platform as a Service $(\mathrm{PaaS})$, and Software as a Service (SaaS) respectively [1]. Clouds have various elements using enormous energy, thus thermal and energy management are the major issues of cloud computing system due to aggregation of computing, networking, and storage hardware, the energy consumption required to transport the data from and to the user constitute [2]. Increasing popularity and demand for cloud services led to growing more large scale data centers with high operating cost, huge amount of energy and emit considerable carbon dioxide. Research shows that, the Information and Communication Technologies (ICT) industry produce $\% 2$ of global $\mathrm{CO}_{2}$ emission which is equal to the aviation industry [3], and it is increasing at a rate of $6 \%$ per year and with such a growth rate they could represent $12 \%$ of worldwide emissions by 2020 [4] and a decrease in emission volume of $15-30 \%$ is required before the year 2020 to keep the global temperature increase below $2^{\circ} \mathrm{C}$ [5]. Also Human influences on global climatic change and threat of fossil fuel depletion have increased sociological movements toward adopting more energy-efficient and ecologically friendly lifestyles during the past three decades [6]. International Data Corporation (IDC) in September 2008 [4], indicate that almost half of 459 European companies surveyed had put in place a strategy for incorporating green IT and cost saving as main drivers for going green. According to a research done by Accenture, moving business applications to cloud can reduce carbon footprint of organizations, which small businesses saw the most dramatic reduction in emissions - up to 90 percent while using cloud resources and the large corporations can save at least 30-60 percent in carbon emissions using cloud applications, and mid-size businesses can save 60-90 percent [7]. The data center dynamics 2012 Global Census states that total power consumption of data centers increased globally from $24 \mathrm{GW}$ to $38 \mathrm{GW}$ (63\%) between 2011 and 2012 [8].

The organization of the paper is as follows. Section 2 gives a brief review of related works. In Section 3, some metrics to evaluate efficiency have been introduced which are widely used. In section 4, the proposed approach are presented to solve the carbon and energy-aware allocation problem. Section 5 describes the simulation design and the evaluation of proposed approach. Section 6 presents the conclusion and future work.

\section{RELATED WORK}

Numerous studies have been done by researchers in recent years and study on power consumption and green computing on various aspects, some of them has been cited here.

Si-Yuan Jing and et al addressed the issue of energy consumption in cloud computing and reviewed some available energy efficient techniques at infrastructure level such as CPU, server, network, storage and cooling infrastructure. They propose a set of feasible solutions in their work [5]. Anton Beloglazov and et al proposed an architectural framework and principles for energy-efficient cloud computing and based on, they present the vision, challenges, resource provisioning and allocation algorithms to gain an energy-efficient management in cloud computing environments [1]. In another study by Saurbh Kumar Garg and et al, a carbon-aware green framework has been proposed, 
which addresses the environmental problem and aim to reduce the emitted carbon footprint by cloud computing [9]. Fereydoun Farahi Moghaddam and et al have studied about low carbon private cloud and focused on VM migration in WAN [10]. The most similar work to this paper is [3] which Atefeh Khosravi and et al proposed an algorithm named "ECE" that considering the carbon emission and power usage effectiveness (PUE) of distributed data centers, In contrast to ours the VM placement problem had seen as a bin-packing problem. Nguyen Quang-Hung and et al [11] proposed a genetic algorithm for power-aware scheduling of resource allocation (GAPA) in order to solve the static VM allocation problem (SVMAP). In 2013, Amritpal Kaur and et al proposed an approach to reduce the carbon impact and power consumption in the data center by considering the green factor of data centers, concepts of cloud computing and its core services have been discussed, also a survey of different energy saving techniques for efficient usage of resources has been done [12]. Toni Mastelic and et al in 2014 performed a comprehensive analysis of an infrastructure supporting the cloud computing paradigm with regards to energy efficiency. Their survey focused on energy efficiency of ICT equipment such as servers and networks, and software solutions running on top of ICT equipment such as Cloud Management System (CMS) and appliance [13]. In another study done by D.Kumar and et al, a genetic algorithm framework has been proposed for task scheduling to minimize the energy consumption in cloud computing infrastructure [14].

F.Kong and X.Liu [15] investigates the green-energy-aware power management problem for modern data centers which integrate renewable or green energy sources into their power supply and surveys and classifies works that explicitly consider renewable energy and/or carbon emission. They group green-energy-aware works into four categories as follows, Green-energy-aware workload scheduling, Greenenergy-aware Virtual Machine management, Green-energyaware energy capacity planning and Interdisciplinary. These categories may be further divided into subcategories according to the number of involved data centers. By this classification this work will be placed in Green-energy-aware workload scheduling with geo-distributed data centers. In another survey study by Fahimeh Alizadeh Moghaddam and et al 2015, state of the art in energy-efficient networking solutions in cloud-based environments reviewed and showed that the decision framework is the most frequently investigated solution type to accomplish the energy efficiency goal [8]. Dang Minh Quan and et al [16] in 2012 proposed an algorithm to find a server in the data center with the lowest energy consumption and/or carbon emission and move the workload there. The algorithm is used for resources management in a federated data center. In 2009, Liang Liu and et al [17], proposed GreenCloud architecture to reduce data center power consumption and enables onlinemonitoring, live virtual machine migration and VM placement optimization by consolidation the workload. GreenCloud consist of several components such as Monitoring Service, Migration Manager and Managed Environment. Another power-efficient resource provisioning technique in cloudbased data centers proposed by [18] in 2013. Since optimization of power-efficient virtual network provisioning is NP-hard, they propose a heuristic algorithm in cloud-based data centers. GreenSlot [19] is a parallel batch job scheduler for a datacenters which partially powered by solar energy and schedules the use of green energy in a greedy manner in datacenters to lower brown energy consumption, monetary costs, and environmental impact. Peter Xiang Geo And et al
[20] proposed FORTE (Flow Optimization based framework for request-routing and traffic engineering) to control the three-way tradeoff between average job time, electricity cost, and carbon emissions. In another study done by Federico Larumbe and Brunilde Sanso [21], a cloud network planning problem presented and a model proposed that allowed planners to evaluate different solutions and to make variations in the optimization priorities.

\section{BACKGROUND}

\subsection{Cloud Broker Definition}

According to the National Institute of Standards and Technology (NIST) [22], "A cloud broker is an entity that manages the use, performance, and delivery of cloud services and negotiates relationships between cloud providers and cloud consumers". The cloud broker acts as the intermediate between consumer and provider and will help consumers through the complexity of cloud service offerings and may also create value-added cloud services as well [22]. Figure 1 depicts the usage scenario for cloud brokers [22].

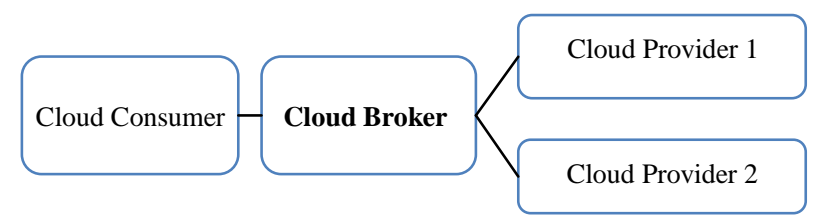

Fig 1: Usage Scenario for Cloud Brokers [22].

\subsection{Power usage effectiveness (PUE)}

PUE is one of the most famous metrics used to measure energy efficiency of a cloud computing services and it is defined as the total power used by the data center $(\mathrm{Pt})$ divided by the total power consumed by the ICT equipment (Ps), and it can defined by Equation 1 [6].

$$
P U E=\frac{P_{t}}{P_{s}}
$$

The ideal value for PUE is 1 if all of the power consumed by the servers account for all of the power delivered to the data center and it is impossible to have a PUE less than 1 [6]. Data from Uptime Institute surveys suggests that average PUE of today's data centers is between 1.8 and 1.89 [6]. In [23], the authors benchmarked 22 data center buildings on 22 data center, which reported that the PUE values between 1.33 and 3 , and the average value was 2.04 . According to the report to congress on servers and data center energy efficiency, PUE ratio of 2.0 was assumed to be the average value across all U.S. data centers [24]. Levels of energy efficiency based on PUE are presented in [25].

A PUE value equal to or close to 1 is theoretically possible by spending zero energy on cooling. The near-zero cooling energy is possible by using free environmental cold-air-, water-, and evaporation-based cooling economizers such as in the Facebook data center [26].

\subsection{Carbon usage effectiveness (CUE)}

For such data centers which get their entire power from electric power distribution and do not leave the local carbon footprints, CUE is defined as equation 2 [27]. In contrast to PUE which is expressed as a number without unit, the units of the CUE metric are kilograms of carbon dioxide ( $\mathrm{kgCO} 2 \mathrm{eq})$ per kilowatt-hour $(\mathrm{kWh})$

$C U E=C E F \times P U E$ 


\section{PROPOSED APPROACH}

\subsection{Roles}

The proposed approach which named "two phase carbon aware cloud broker" aim to reduce the carbon footprint and power consumption in data centers. The proposed approach considers three main roles: user, cloud provider and Green Cloud Broker.

User: Users requested the execution of their cloud tasks (cloudlet) to the broker with an estimated length. Every cloudlet has a length which means the number of its internal Instructions and the unit is Million Instructions (MI).

Cloud provider: Cloud providers; rent their services on payas-you-go model. In infrastructure as a service (IaaS), they rent their infrastructures; data centers are known as a set of physical machine, each of them has their own resources (CPUs, Memory, and Network, Bandwidth and storage space). Each cloud providers may have one or more data centers allocate in different places with different specifications. Hence every cloud provider is responsible for updating related parameters such as data centers PUE, carbon emission rate and available physical machines.

Green cloud broker: responsibility of green cloud broker is same as a common cloud broker with the difference that green cloud broker is responsible for calculating the carbon footprint from the execution of cloudlets. In the first phase, by using the information in the catalog which has been received from cloud providers, assigns the VMs to physical servers in a data centers (may be different). In the second phase, by considering the length of cloudlets, VM requested specification and it's deadline, assign cloudlet to appropriate virtual machine. Also at the end of every scheduling interval, it is responsible for estimating carbon footprints and power consumption to rank the providers and their data centers.

\subsection{Power model}

Computational node's power consumption in the data centers is generally determined by the CPU, memory, disk storage space, and cooling systems [28]. Unlike other systems, in this case more power is being consumed by the processor [1]. Therefore, in this study, only CPU has been considered [1] Showed that even in the case of DVFS is used; there is a linear relation between power consumption and CPU utilization. In this work, Equation 3 [1] is used to calculate the power consumption of servers.

$P(\mathrm{u})=\mathrm{k} \times \mathrm{P}_{\max }+(1-\mathrm{k}) \times P_{\max } \times v$

Where $\mathrm{P}_{\max }$ is the maximum power a server consumes at its full utilization, $\mathrm{k}$ is the percent of the energy that a server uses in the idle state and $u$ is the utilization percent of CPU. CPU utilization may vary according to time and depending on its workload. Energy consumption as a function of time is expressed as $\mathrm{u}(\mathrm{t})$. Therefore, the energy consumption of a physical node can be expressed as an integral power at time $\mathrm{t}$ [5], it shown in Equation 4.

$\int_{0}^{t_{1}} P(u(t)) d_{t}$

This fact and the problem of power consumption model of modern multi-core processors combined and causes exact analytical model be a complex research problem. So instead of using the analytical power of a server, the actual power consumption data published by the SPECpower ${ }^{1}$ benchmark was used.

\subsection{Scenario}

In this scenario, $\mathrm{n}$ VM should be assigned to $\mathrm{m}$ Physical machines and then $\mathrm{p}$ cloudlet should assign to them. As stated before users requested the implementation of their cloud tasks (cloudlet) to the broker with an estimated length, in every scheduling interval, by using the information in the catalogue, broker assigns the VMs to physical servers in data centers, after that in the second phase, by considering the specification cloudlets assign to appropriate virtual machine. If the source of data center`s energy was not clean (such as fossil fuels), there is a direct relationship between power consumption and their carbon footprint. If the energy source is totally clean, no matter how much power is consumed, the carbon resulting would be zero. The relationship between power consumption and carbon is described in the equation 5 [10].

$C_{d}^{p}(t)=\varphi_{d}(t) P_{d}(t)$

Where $\mathrm{C}_{\mathrm{pd}}(\mathrm{t})$ represents the amount of carbon that remains at time $\mathrm{t}$ by the data center d. $\rho_{d}(t)$ represents the exchange rate between power and carbon at time $t$ for data center d. $P_{d}(t)$ represents the power consumption of data center $d$ at time $t$. Each data center can be powered by different sources of energy, and each of these sources has their own carbon footprint [29].

As in equation 6 states, $\rho_{\mathrm{d}}(\mathrm{t})$ depends on the type of data center energy source and especially for data centers which fed by several energy sources it can be vary over time [10]

$$
\varphi_{d}(t)=\frac{\sum \operatorname{source}_{d_{\text {source }}}(t) P_{d_{\text {source }}}(t)}{\sum \operatorname{sourceP}_{d_{\text {source }}}(t)}
$$

\subsection{First phase of proposed approach}

In the first phase, virtual machine assigns to physical servers in a data centers. In this phase, the minimum percent of virtual machine is specified should be created in data centers. Then, according to CUE parameter and maximum load for data center, the remaining virtual machines are deployed on existing data centers. At this point (the second stage of the first phase) data centers with lower CUES have a higher priority. In this way virtual machine would be created on the data centers. Figure 2 shows this phase as a pseudo code.

Algorithm 1: CreateVmsInData center

1. Input: Data center List, VmList

2

3. Sort Data centerList by CUE;

4. VmList $\leftarrow$ get the vms has been requested

5. Data centerList $\leftarrow$ get available Data centers

6.

7. For $\mathrm{DC}_{\mathrm{i}} \in\{$ Data centerList $\}$

8. int $\mathrm{DC}_{\mathrm{i}}$.allocated $\mathrm{Vm}=0$;

9. While $\left(\mathrm{DC}_{\mathrm{i}} \cdot \mathrm{MinVM}>\mathrm{DC}_{\mathrm{i}}\right.$.allocated $\left.\mathrm{Vm}\right)$

10. Send vm to data center

${ }^{1}$ The SPECpower benchmark results for the fourth quarter of 2010.http://www.spec.org/power_ssj2008/results/res201 $0 \mathrm{q} 4 /$ 


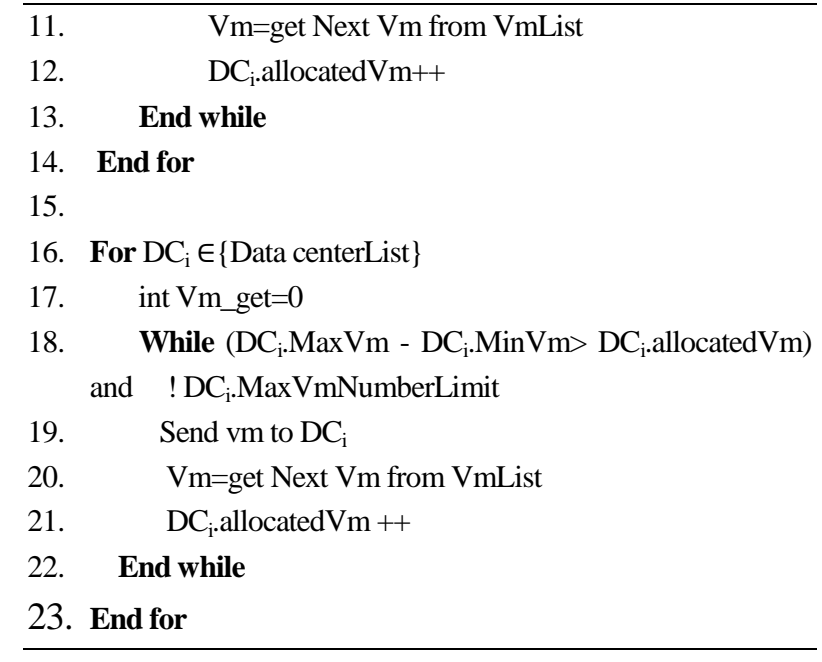

Fig 2: Pseudo-code for the first phase

\subsection{Second phase of proposed approach}

The purpose of this phase is assignment of cloudlets on virtual machines and reduces the carbon footprint and energy consumption. For the second phase, Genetic Algorithm has been used and the proposed fitness function is based on the carbon footprint due the running of cloudlets on the virtual machines is measured. And the goal is minimizing this amount. At the beginning each cloudlets has a specified length in terms of millions of instructions. Each virtual machine also has a processing speed based on millions of instructions per second (MIPS). The time of cloudlets can be calculated on a virtual machine. Also due to the virtual machine on which the data center is located, it can be calculated that the carbon footprint of the energy performance of a cloudlets what endures. Fitness function used in this phase is according to equation 7.

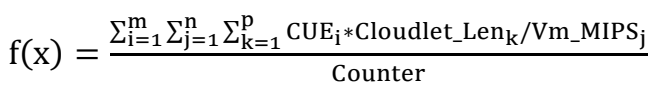

Where, $\mathrm{P}$ is the number of cloudlets, $\mathrm{n}$ is the number of virtual machines, $m$ is the number of data centers which $m>n$ and counter is the number of Host underutilization. Figure 3 show the second phase of the proposed approach which described as pseudo code.

\begin{tabular}{ll}
\hline \multicolumn{2}{l}{ Algorithm $2:$ SubmitCloudlets } \\
\hline 1. & Input: Data centerList, VMlist, CloudletList \\
2. & \\
3. & Data centerList $\leftarrow$ get available Data centers \\
4. & VMList $\leftarrow$ get the vms has been requested \\
5. & CloudletList $\leftarrow$ get the Cloudlet List has been requested \\
6. & \\
7. & Set ChromSize $=$ Number of Cloudlets \\
8. & Set ChromData $=$ Number of VMs; \\
9. & Set Fitness Function \\
10. & Set Population Size=VMsList.size /Data centerList.size \\
11. & Create random chromosomes \\
12. & Start Genetic Algorithm \\
13. & Get Best Chromosome \\
14. & \\
15. & For Cloudlet $\in$ \{CloudletList $\}$ \\
\hline
\end{tabular}

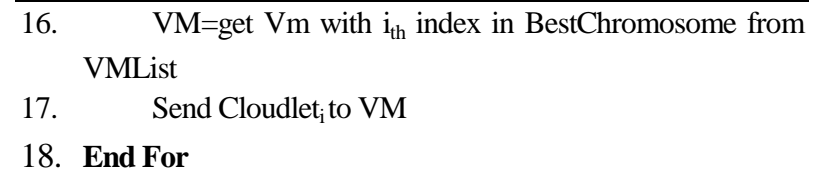

Fig 3: Pseudo-code for the Secend phase

The cloudlet_lenght is a property of cloudlet which defined as number of Instructions and it's unit is Million Instructions (MI).

\section{SIMULATION}

The simulations have been performed by extending Cloudsim3.02 [30] in Netbeans. To simulate the proposed approach a scenario has been defined with four data centers in different location has been considered [3], they are presented in Table 1. Each data center includes 100 physical servers. Specifications of physical servers and virtual machines are presented in Table 2 and Table 3 respectively.

Table 1. Data center Characteristics [3]

\begin{tabular}{|c|c|c|}
\hline Data center Site & PUE & $\begin{array}{c}\text { Carbon Footprint } \\
\text { Rate (Tons/MWh) }\end{array}$ \\
\hline DC1 -Oregon, USA & 1.56 & $0.124-0.147$ \\
\hline DC2 -California, USA & 1.7 & $0.350-0.658$ \\
\hline DC3 -Virginia, USA & 1.9 & $0.466-0.782$ \\
\hline DC4 -Dallas, USA & 2.1 & $0.678-0.730$ \\
\hline
\end{tabular}

Table 2. Characteristics Of Virtual Machine

\begin{tabular}{|c|c|c|}
\hline Virtual Machine & TYPE A & TYPE B \\
\hline Number of cores & 1 & 1 \\
\hline $\begin{array}{c}\text { Processing speed } \\
\text { (MIPS) }\end{array}$ & 500 & 1000 \\
\hline Memory RAM (MB) & 1740 & 2048 \\
\hline Storage space(GB) & 2.5 & 2.5 \\
\hline
\end{tabular}

Type of applications is considered as bag-of-tasks and the arrival time of requests generates by exponential distribution. Initial population was generated random and maximum generation size. The probability mutation has been set to 0.01 and size of population is 10 [11]. Limited load balancing for all data center is set to be equal to at least $20 \%$ and a maximum of $90 \%$ is set. Six different workloads generated based on an exponential distribution with mean values = $\{50000, \quad 70000,100000,120000,150000,200000\}$ respectively named \{workload_0, workload_1,..., workload_6\}.

The simulations performed with the settings mentioned before. To achieve a normal value, each experiment is repeated 30 times and the mean value is reported. The result of the proposed method was compared with Round-Robin algorithm [31].The result of the simulations shows that by using the proposed method, energy consumption and carbon footprint has been improved.

Simulation results are shown in Figure 4 and 5 respectively. The energy consumption improvement and carbon footprint improvement shown in Fig 6 and 7 respectively.

Fig 8 depicts the comparison of carbon footprint in Data Centers between proposed method and Round-Robin. 
Table 3. Characteristics Of Physical Machine

\begin{tabular}{|c|c|c|}
\hline & $\begin{array}{c}\text { HP PROLIANT } \\
\text { ML110 G5 }\end{array}$ & $\begin{array}{c}\text { HP PROLIANT } \\
\text { ML110 G4 }\end{array}$ \\
\hline Server & Intel Xeon 3075 & Intel Xeon 3040 \\
\hline Processor name & 500 & 1000 \\
\hline Cores & 2 & 2 \\
\hline $\begin{array}{c}\text { Processor } \\
\text { frequency }\end{array}$ & 2660 & 1860 \\
\hline
\end{tabular}
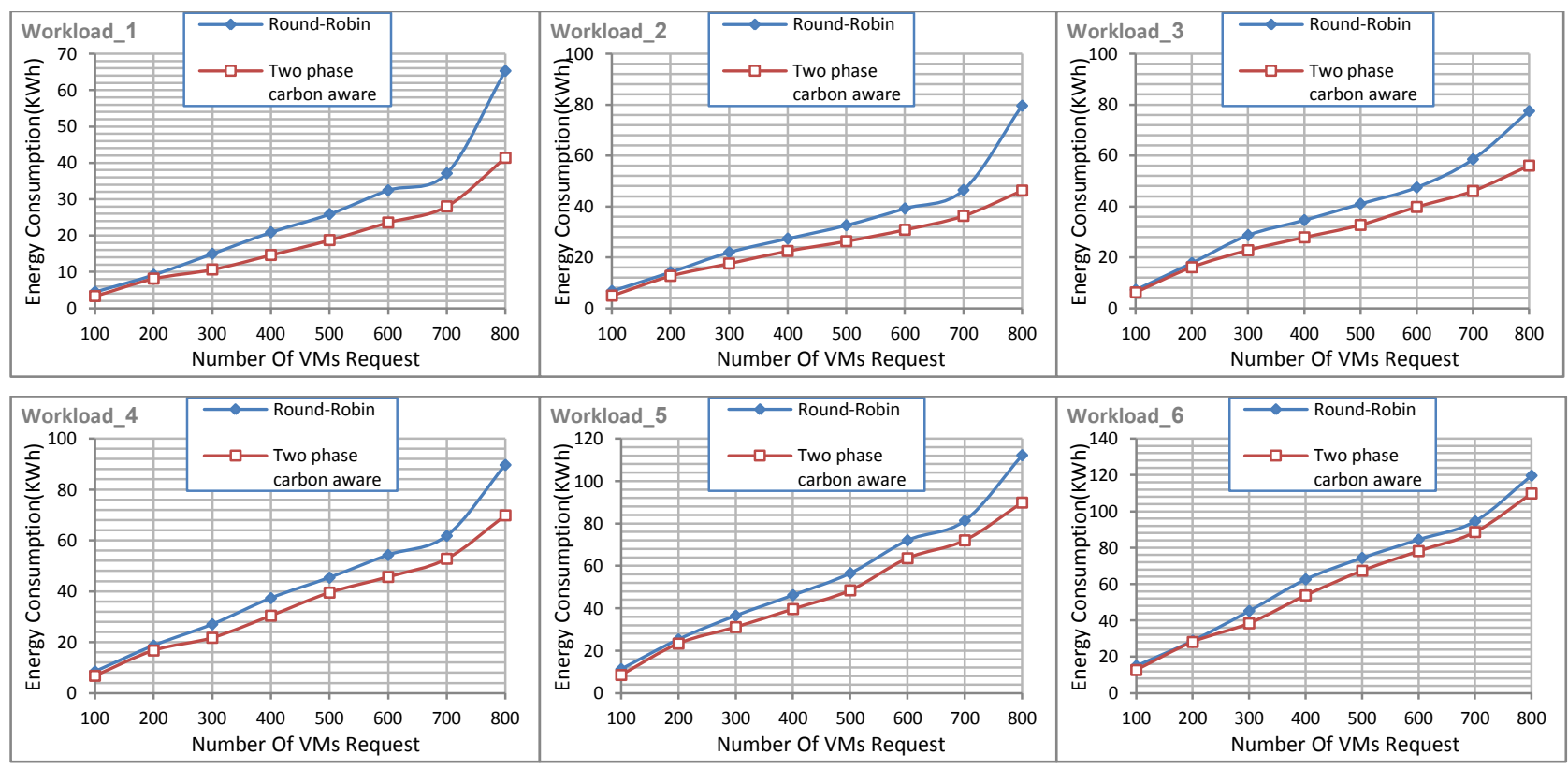

Fig 4: Comparison of energy consumption between "Two Phase Carbon Aware" and Round-Robin

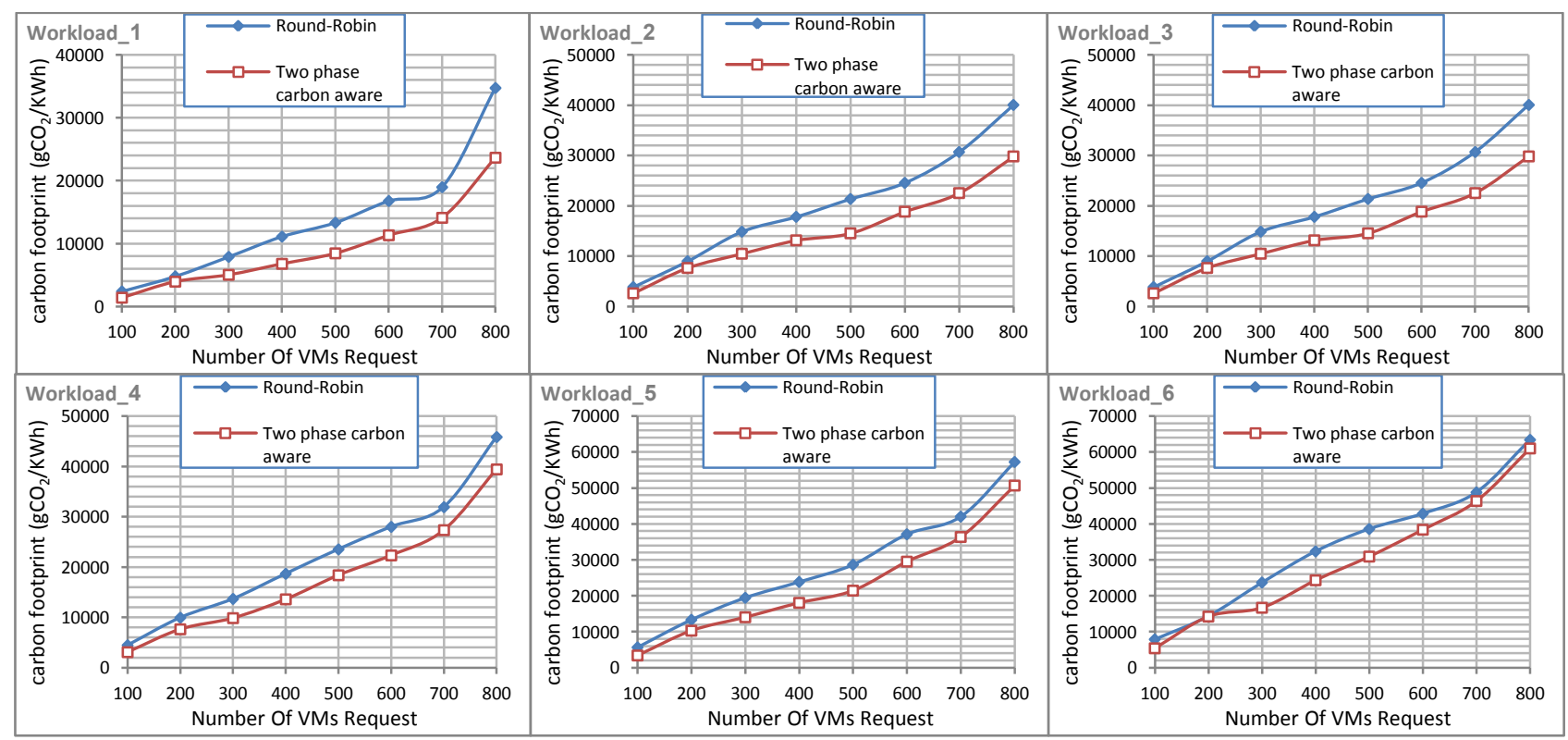

Fig 5: Comparison of carbon footprint between "Two Phase Carbon Aware" and Round-Robin 


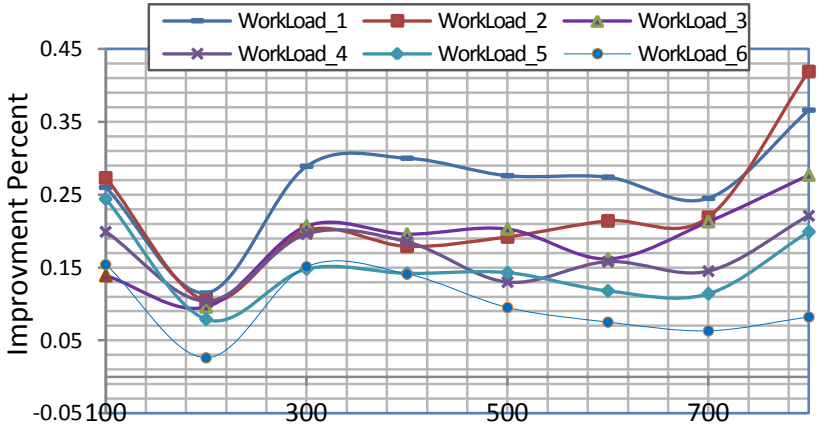

Number Of VMs Request

Fig 6: Energy consumption improvement in "Two Phase Carbon Aware" in difference with Round-Robin
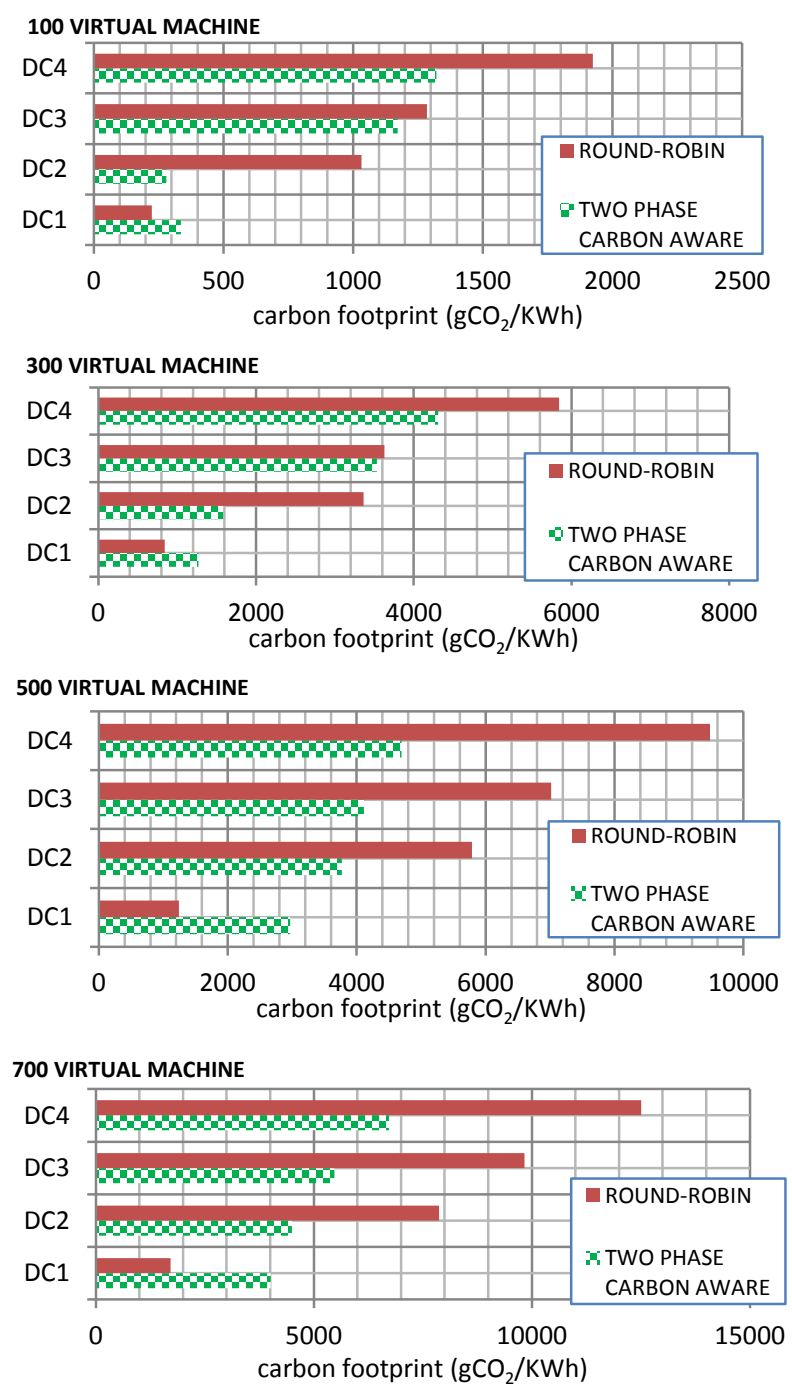

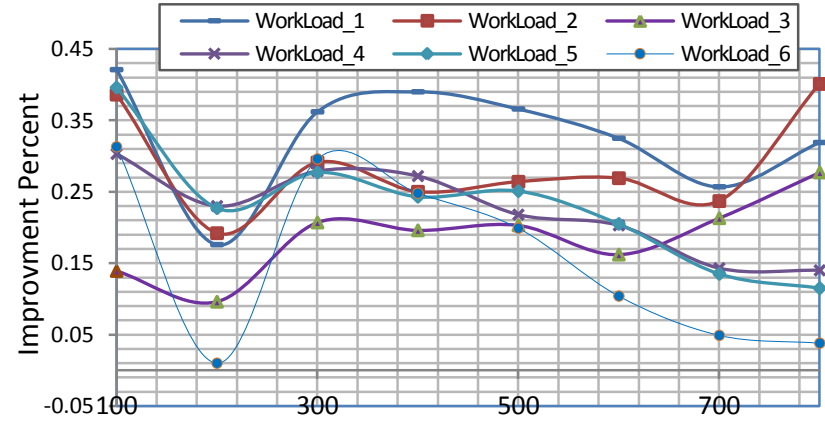

Number Of VMs Request

Fig 7: Carbon footprint improvement in "Two Phase Carbon Aware" in difference with Round-Robin

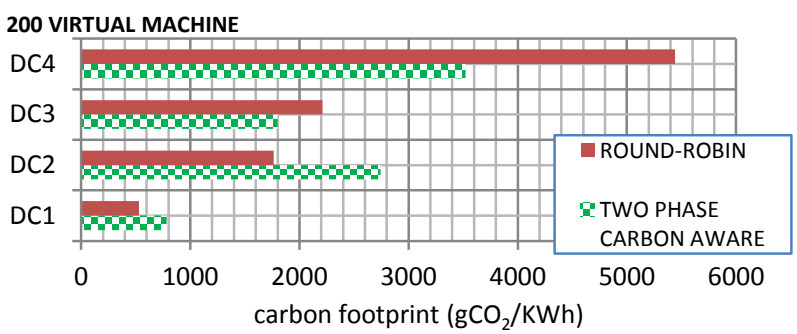

400 VIRTUAL MACHINE

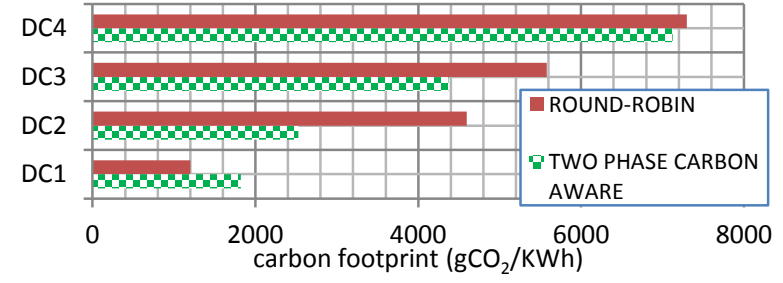

600 VIRTUAL MACHINE

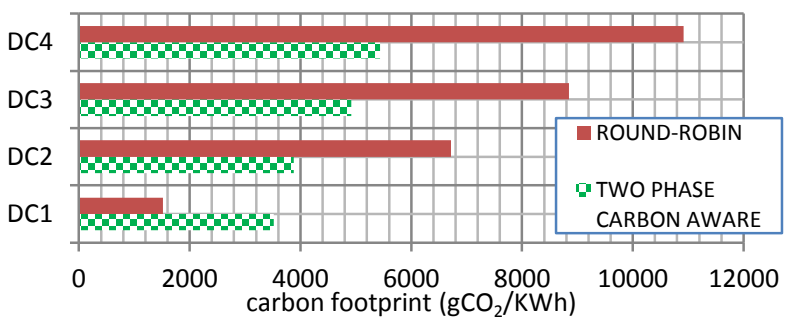

800 VIRTUAL MACHINE

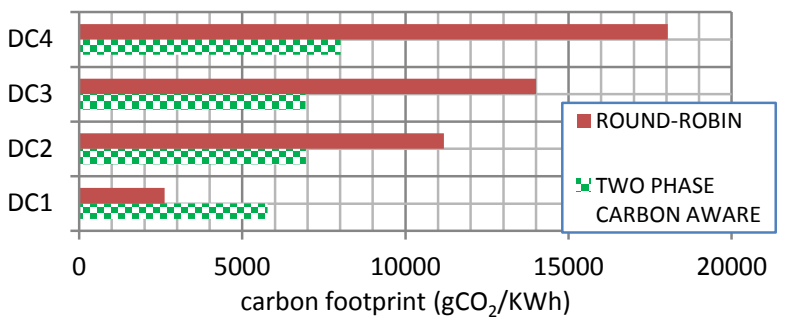

Fig 8: Comparison of carbon footprint between "Two Phase Carbon Aware" and Round-Robin

\section{CONCLUSION AND FUTURE WORK}

In this paper, by considering the central role of the Cloud Broker in cloud computing, "Two Phase Carbon Aware Cloud Broker" has been proposed that attempt to minimize energy and carbon by considering the energy and carbon efficiency of data centers which may geographically distributed. For developing the "Two Phase Carbon Aware Cloud Broker", Genetic algorithm has been used to select and place the cloudlets on proper VM.

In order to do simulation-based evaluation, CloudSim has been extended. Simulation results demonstrate that the proposed approach can reduce the carbon emission and energy 
consumption up to $20 \%, 15 \%$ in comparison to Round Robin respectfully.

In the future, trade of between parameters such as SLA, revenue of service providers and cost of energy, Should be considered. Also in order to achieve green cloud computing and improving energy consumption, live VM migration policies should be reviewed.

\section{REFERENCES}

[1] A.Beloglazov, J.Abawajy, R.Buyyaa, "Energy-aware resource allocation heuristics for efficient management of data centers for Cloud computing," Future Generation Computer Systems, no. 28, pp. 755-768, 2012.

[2] S.Patil, P.Pattenshetti, "Overview of Green Cloud Architecture," International Journal of Computer Applications, pp. 9-12, 2014

[3] A.Khosravi, S.K.Garg, R.Buyya, "Energy and Carbon Effcient Placement of Virtual Machines in Distributed Cloud Data Centers," 2013.

[4] M.Uddin, A.Rahman, "Energy efficiency and low carbon enabler green IT framework for data centers considering green metrics," Renewable and Sustainable Energy Reviews, vol. 16, no. 6, pp. 4078-4094, August 2012.

[5] S.Jing, Sh.Ali, K.She, Y.Zhong, "State-of-the-art research study for green cloud computing," The Journal of Supercomputing, December 2011.

[6] J.Yuventi, R.Mehdizadeh, "A critical analysis of Power Usage Effectiveness and its use incommunicating data center energy consumption," Energy and Buildings, vol. 64, pp. 90-94, 2013.

[7] S.Murugesan, G.Gangadharan, "Green Cloud Computing and Environmental Sustainability" UK: Wiley Press, 2012, pp. 315-340.

[8] F.A.Moghaddam, P.Lago, P.Grosso, "Energy-Efficient Networking Solutions in Cloud-Based Environments: A Systematic Literature Review," ACM Computing Surveys, vol. 47, May 2015.

[9] S.K.Garg, C.S.Yeo, R.Buyya, "Green Cloud Framework for Improving Carbon Efficiency of Clouds," in Euro-Par 2011 Parallel Processing. Bordeaux, France: Springer Berlin Heidelberg, 2011, pp. 491-502.

[10] F.F.Moghaddam, M.Cheriet, K.K.Nguyen, "Low Carbon Virtual Private Clouds," in IEEE 4th International Conference on Cloud Computing, 2011, pp. 259-166.

[11] N.Quang-Hung, P.Dac Nien, N.Hoai Nam, N.Huynh Tuong, N.Thoai, "A Genetic Algorithm for Power-Aware Virtual Machine Allocation in Private Cloud," Information and communication technology lecture notes in computer science, vol. 7804, pp. 183-191, 2013.

[12] A.Kaur, S.Kinger, "Increasing Cloud Usage: A Shift towards, Green Clouds" International Journal of Computer Applications, vol. 67, pp. 28-32, April 2013.

[13] T.Mastelic, A.Oleksiak, H.Claussen, I.Brandic, J.Pierson, A.V.Vasilakos, "Cloud Computing: Survey on Energy Efficiency," ACM Computing Surveys, vol. 47, 2014.

[14] D.Kumar, B.Sahoo, B.Mondal, T.Mandal, "A Genetic Algorithmic approach for Energy Efficient," International Journal of Computer Applications, vol. 118, pp. 1-6, May 2015.
[15] F.Kong, X.Liu, "A Survey on Green-Energy-Aware Power Management for Datacenters", ACM Computing Surveys. Vol. 47, No. 2, Article 30, 2014

[16] D.M.Quan, A.Somov, C.Dupont, "Energy Usage and Carbon Emission Optimization Mechanism for Federated Data Centers," in Energy Efficient Data Centers. Madrid, Spain: Springer Berlin Heidelberg, 2012, pp. 129-140.

[17] L.Liu, H.Wang, X.Liu, X.Jin, W.He, Q.Wang, Y.Chen, "GreenCloud: A New Architecture for Green Data Center," in 6th International Conference IndustrySession on Autonomic Computing and Communications Industry Session (ICAC-INDST'09), New York, 2009, pp. 29-38.

[18] G.Sun, V.Anand, D.Liao, C.Lu, X.Zhang, N.Bao, "Power-Efficient Provisioning for Online Virtual Network Requests in Cloud-Based Data Centers," Systems Journal, vol. 9, no. 2, pp. 427 - 441, 2013.

[19] I.Goiri, K.Le, M.E.Haque, R.Beauchea, T.D.Nguyen, J.Guitart, J.Torres, R.Bianchini, "GreenSlot: Scheduling Energy Consumption in Green Datacenters," in International Conference for High Performance Computing, Networking, Storage and Analysis, Seattle, 2011, pp. 1-11

[20] P.X.Gao, A.R.Curtis, B.Wong, and S. Keshav, "It's Not Easy Being Green," ACM SIGCOMM Computer Communication Review - Special october issue SIGCOMM '12, vol. 42, no. 4, pp. 221-222, October 2012.

[21] F. Larumbe, B.Sanso, "A Tabu Search Algorithm for the Location of Data Centers and Software Components in Green Cloud Computing Networks," IEEE Transactions on Cloud Computing, vol. 1, no. 1, pp. 22-35, JANUARY-JUNE 2013

[22] F.lue, J.Tong, J.Mao, R.Bohen, J.Messina, L.Badger, D.Leaf. (2011, september) Nist. [Online]
[One www.nist.gov/customercf/get_pdf.cfm?pub_id=909505

[23] S.Greenberg, E.Mills, B.Tschudi, P.Rumsey, B.Myatt, "Best practices for data centers: lessons learned from benchmarking 22 data centers," ACEEE summer study on energy efficiency in buildings. PacificGrove, 2006.

[24] "Report to Congress on Server and datacenter energy efficiency Public law," US Environmental Protection Agency, Energy Star Program August 2, 2007.

[25] J.Choa, T.Limb, B.S.Kimb, "Viability of datacenter cooling systems for energy efficiency in temperate or subtropical regions: Case study," Energy and Buildings, no. 55, pp. 189-197, 2012

[26] M.T.Chaudhry, T.C.Ling, A.Manzoor, S.A.Hussain, J.Kim, "Thermal-Aware Scheduling in Green Data Centers," ACM Computing Surveys, vol. 47, February 2015.

[27] C.Belady, D.Azevedo, M.Patterson, J.Pouchet, R.Tipley. (2010) http://www.thegreengrid.org/. [Online] http://www.thegreengrid.org/ /media/WhitePapers/Carb on\%20Usage\%20Effectiveness\%20White\%20Paper_v3. pdf?lang=en

[28] L.Minas, B.Ellison, "Energy Efficiency for Information Technology: How to Reduce Power Consumption in Servers and Data Centers," Intel Press, 2009. 
[29] M.Lenzen, "Current State of Development of ElectricityGenerating Technologies," A Literature Review Energies, no. 3, pp. 462-591, 2010.

[30] R.Buyya, R.Ranjan, R.N.Calheiros, "Modeling and simulation of scalable cloud computing environments and the cloudsim toolkit: Challenges and opportunities," in High Performance Computing \& Simulation
HPCS0'09. International Conference IEEE, 2011, pp. 111.

[31] J.Li, M.Qiu, Z.Ming, G.Quanc, X.Qin, Z.Gue, "Online optimization for scheduling preemptable tasks on IaaS cloud systems," Journal of Parallel Distributed Computing, pp. 666-677, 2012. 